PROCEEDINGS OF THE

AMERICAN MATHEMATICAL SOCIETY

Volume 131, Number 4, Pages 1115-1120

S 0002-9939(02)06922-8

Article electronically published on November 13, 2002

\title{
A PRIORI ESTIMATES FOR QUASILINEAR DEGENERATE PARABOLIC EQUATIONS
}

\author{
MARIA MANFREDINI AND ANDREA PASCUCCI
}

(Communicated by David S. Tartakoff)

\begin{abstract}
We prove some maximum and gradient estimates for classical solutions to a wide class of quasilinear degenerate parabolic equations, including first order ones. The proof is elementary and exploits the smallness of the domain in the time direction.
\end{abstract}

\section{INTRODUCTION}

We aim to show some simple a priori estimates for solutions to a quasilinear degenerate equation of the form

$$
P u \equiv-u_{t}+\sum_{i, j=1}^{N} a_{i j}(X, u, D u) D_{i j} u+a(X, u, D u)=0,
$$

where $\left.X=(x, t) \in Q_{T} \equiv \Omega \times\right] 0, T\left[\right.$ and $\Omega$ is a bounded domain of $\mathbb{R}^{N}$. As usual, $u_{t}, D_{i j} u$ stand for $\partial_{t} u, \partial_{x_{i} x_{j}} u$ respectively and we denote by $D=D_{x}$ the gradient with respect to the spatial variables. The coefficients $a_{i j}(X, z, p), i, j=$ $1, \ldots, N, a(X, z, p)$ are assumed to be defined for all values of $(X, z, p)$ in $Q_{T} \times \mathbb{R} \times \mathbb{R}^{N}$. Besides the principal matrix $A=\left(a_{i j}\right)$ is supposed symmetric and positive semidefinite (the case $A=0$ of a first order equation is included).

In many contexts the obtainment of bounds for the solution and its gradient plays a crucial role. It is well known that the solvability of a boundary value problem for a parabolic or elliptic equation can be reduced to the establishment of a priori estimates of solutions. In the degenerate parabolic case, the equation is often regularized by adding a vanishing diffusion term as follows:

$$
P_{\varepsilon} \equiv P+\varepsilon \Delta_{x}, \quad \varepsilon>0,
$$

where $\Delta$ denotes the Laplacian operator in the spatial variables. Consequently it is important to obtain $\varepsilon$-uniform estimates of solutions, namely estimates which are independent on the "parabolicity" constant of the equation. Gradient estimates are normally achieved by ad hoc arguments which are, in most cases, modifications of the classical Bernstein method [3]. More precisely, under suitable hypotheses, by differentiating the equation it is possible to obtain estimates by means of the maximum principle and classical barrier functions arguments. In general, these estimates are only valid if the domain is sufficiently small in the time direction,

Received by the editors October 15, 2001.

2000 Mathematics Subject Classification. Primary 35K55; Secondary 35K65.

This work was supported by the University of Bologna, funds for selected research topics. 
due to the occurrence of blow up phenomena. This is the case, for instance, of the Burgers' equation in $\mathbb{R} \times \mathbb{R}^{+}$

$$
-u_{t}+u \partial_{x} u=0
$$

or the well known semilinear heat equation

$$
-u_{t}+\Delta u+u^{p}=0, \quad p>1 .
$$

On the other hand, by exploiting the smallness of $T$ in (1.1), it is possible to obtain estimates of the solution even if the operator $P$ does not satisfy a standard comparison principle. Indeed in this paper we aim to remark that the fact that $T$ is sufficiently small is almost sufficient to obtain at least maximum estimates and, in many cases, gradient bounds. This fact was implicitly used by Pascucci and Polidoro [14] and then by Citti and Manfredini [5] to study some non-linear degenerate equations with applications to finance and image processing. Here we aim to show the following a priori estimates which, to the best of our knowledge, have not appeared in print. Our first result is the following

Theorem 1.1 (Maximum estimates). Assume that the function $a(\cdot, \cdot, 0)$ is bounded on $Q_{T} \times K$ for every $K$ bounded subset of $\mathbb{R}$. Suppose that $u \in C^{2}\left(Q_{T}\right)$ is such that

$$
\left\{\begin{array}{l}
P u \geq 0 \quad \text { in } Q_{T}, \\
\limsup _{\mathcal{P} Q_{T}} u \leq C,
\end{array}\right.
$$

where $C \geq 1$ and $\mathcal{P} Q_{T}$ denotes the parabolic boundary of the cylinder $Q_{T}$. Then there exists $\left.\left.T_{0} \in\right] 0, T\right]$, which depends only on $\Omega, a(\cdot, \cdot, 0)$ and $C$, such that

$$
\sup _{Q_{T_{0}}} u \leq 3 C .
$$

We remark that $T_{0}$ in Theorem 1.1 does not depend on $\left(a_{i j}\right)$, therefore (1.6) provides an $\varepsilon$-uniform estimate for the regularized equation (1.2).

As a consequence of Theorem 1.1 we establish some gradient bounds by the Bernstein method. Several extensions of this technique are known in literature. In the case of parabolic equations, considerable generalizations of this method are due to Ladyzhenskaya and Ural'tseva [10, 11, and Serrin [15]. A wide survey of the related literature can be found in Lieberman [12]. We also quote the papers by Ivanov [9] and by Lieberman [13] for the degenerate parabolic case, and by Barles [2] who generalized the Bernstein method to a class of fully non-linear elliptic equations in the framework of viscosity solutions.

Since the Bernstein technique involves the differentiation of equation (1.1), we need to impose some extra structural conditions on the derivatives of the coefficients of $P$. Preferring straightforwardness to generality, in the following theorem we assume the solution $u \in C^{3}$ and the coefficients $a_{i j}, a \in C^{1}$ (see Remark 2.1). Moreover we assume that at least one of the following conditions holds:

(H.1) the principal coefficients $a_{i j}$ can be written as follows:

$$
a_{i j}(X, z, p)=a_{i j}^{*}(p)+\frac{1}{2}\left(p_{i} c_{j}(X, z, p)+p_{j} c_{i}(X, z, p)\right), \quad i, j=1, \ldots, N,
$$

where $a_{i j}^{*}, c_{i} \in C^{1}, i, j=1, \ldots, N$, and the matrix $\left(a_{i j}^{*}\right)$ is positive semi-definite;

(H.2) the matrix $A$ is diagonal and there exist two positive constants $k, M$ such that, for every $i=1, \ldots, N$,

$$
\left|D_{x, z} a_{i i}(X, z, p)\right|^{2} \leq M a_{i i}(X, z, p) \quad \text { whenever } \quad a_{i i}(X, z, p) \leq k .
$$


In (1.8), $D_{x, z}$ stands for $\left(D, \partial_{z}\right)$. As we shall see in the proof, these conditions can be further weakened or adapted to more general settings.

Theorem 1.2 (Gradient estimates). Assume that the functions $D_{x, z} a_{i j}$ and $D_{x, z} a$ are bounded on $Q_{T} \times K$ for every $K$ bounded subset of $\mathbb{R} \times \mathbb{R}^{N}$. If the principal coefficients of the operator $P$ in (1.1) verify condition (H.1) or (H.2), then for every $C \geq 1$ there exists $\left.\left.T_{0} \in\right] 0, T\right]$, which depends on $D_{x, z} a_{i j}, D_{x, z} a, \Omega, k, M$ and $C$, such that for every $u \in C^{3}\left(Q_{T_{0}}\right)$ solution of (1.1) verifying

$$
\underset{\mathcal{P} Q_{T_{0}}}{\lim \sup }|D u|^{2} \leq C
$$

we have

$$
\sup _{Q_{T_{0}}}|D u|^{2} \leq 3 C
$$

We close the Introduction by quoting some examples of applications of our results.

Condition (1.8) is satisfied if $\left(a_{i j}\right)$ in (1.1) is uniformly positive definite. In this case it suffices to choose $k<\inf \lambda$, where $\lambda$ is the smallest eigenvalue of the matrix $\left(a_{i j}\right)$. The following simple equation in $\mathbb{R}^{2}$,

$$
|u|^{\alpha} \partial_{x x} u-\partial_{t} u=0
$$

verifies (1.8) if $\alpha=2$ but not if $\alpha=1$.

Condition (1.7) is satisfied if $\left(a_{i j}\right)$ only depends on $p$. Then obviously $a_{i j}^{*}=a_{i j}$ and $c_{i}=0$. We also refer to Gilbarg and Trudinger 7], Chap. 14, for other examples of such decompositions. In particular, Theorem 1.2 applies to equations (1.4), (1.3) and, in general, to first order equations. Other particular cases are the equation of motion by mean curvature (see, for example, Huisken [8]) and the nonlinear ultraparabolic equation arising in mathematical finance

$$
\partial_{x x} u+u \partial_{y} u-\partial_{t} u=f, \quad(x, y, t) \in \mathbb{R}^{2} \times \mathbb{R}^{+},
$$

studied in [6], 1], 14] and 4]. Theorems 1.1] and 1.2 were also used in [5] to prove a locally existence result for an equation, arising in mathematical vision, of the form

$$
u_{t}=\sum_{i, j=1}^{N} a_{i j}(u) \partial_{x_{i} x_{j}} u+\sum_{i=1}^{N} b_{i}(u) \partial_{x_{i}} u+c(u),
$$

where $u=u(x, y, t),(x, y, t) \in \mathbb{R}^{N} \times \mathbb{R} \times \mathbb{R}$.

\section{Proof of Theorems 1.1 and 1.2}

Throughout this section we adopt the notation

$$
\|f\|_{D}=\sup _{D} f
$$

and we use the summation convention that any term with a repeated index $i$ is summed over $i=1$ to $N$.

Proof of Theorem 1.1. By a standard truncation argument it is not restrictive to suppose that $u \in C(\Omega \times] 0, T])$ and it is positive in some points of $Q_{T}$. Hence $\|u\|_{Q_{T}}$ is finite. Besides we may also assume that

$$
M_{T} \equiv \sup _{Q_{T} \times\left[-\|u\|_{Q_{T}},\|u\|_{Q_{T}}\right]}|a(\cdot, \cdot, 0)|>0 .
$$


We set

$$
v=e^{-t M_{T}} u
$$

By the so-called Weierstrass Lemma, there exists $\bar{z}=(\bar{x}, \bar{t}) \in \bar{Q}_{T}$ such that

$$
\|v\|_{Q_{T}}=\|v\|_{Q_{T} \cap B(\bar{z}, r)}, \quad \forall r>0
$$

where $B(\bar{z}, r)$ denotes the Euclidean ball with radius $r$ and center $\bar{z}$ in $\mathbb{R}^{N+1}$. We may also assume that $\bar{t}<T$.

Then, if $\bar{z} \in \mathcal{P} Q_{T}$, we have

$$
u(z)=e^{t M_{T}} v(z) \leq e^{t M_{T}}\|v\|_{Q_{T}} \leq e^{T M_{T}}\|u\|_{Q_{T} \cap B(\bar{z}, r)} \leq C e^{T M_{T}},
$$

where $C$ is the positive constant in (1.5).

On the other hand, if $\bar{z} \in Q_{T}$, then by assumption $v(\bar{z})$ is strictly positive and

$$
0 \leq P u(\bar{z}) \leq a(\bar{z}, u(\bar{z}), 0)-\partial_{t} u(\bar{z})
$$

(given that $\left.\partial_{t} u(\bar{z})=M_{T} u(\bar{z})\right)$

$$
\leq M_{T}(1-u(\bar{z})) .
$$

Then, by (2.1), $u(\bar{z}) \leq 1$ and also $v(\bar{z}) \leq 1$. Hence, as before, we get $\|u\|_{Q_{T}} \leq e^{T M_{T}}$. In conclusion, we have proved that

$$
\|u\|_{Q_{T}} \leq C e^{T M_{T}}
$$

Next, we set

$$
m_{T} \equiv \sup _{Q_{T} \times[-3 C, 3 C]}|a(\cdot, \cdot, 0)| .
$$

Since $T m_{T}$ tends to zero as $T \rightarrow 0^{+}$, there exists a positive $\left.\left.T_{0} \in\right] 0, T\right]$ such that

$$
e^{t m_{t}} \leq 2, \quad \forall t \in\left[0, T_{0}\right] .
$$

In order to conclude the proof of (1.6), we consider the set $S$ defined as

$$
S=\left\{t \in\left[0, T_{0}\right] \mid\|u\|_{Q_{s}} \leq 3 C, \forall s \in[0, t]\right\} .
$$

Clearly, $S$ is non-empty and closed. The set $S$ is also open; indeed, if $t$ belongs to $S$, then by (2.3), we have

$$
\|u\|_{Q_{t}} \leq C e^{t M_{t}}
$$

(by definition of $S, m_{t}, M_{t}$ )

$$
\leq C e^{t m_{t}} \leq 2 C .
$$

Therefore the thesis follows by continuity. This proves that $S=\left[0, T_{0}\right]$ and concludes the proof of the theorem.

Proof of Theorem 1.2. We differentiate equation (1.1) with respect to the variable $x_{h}$, we multiply the obtained equation by $D_{h} u \equiv \frac{\partial u}{\partial_{x_{h}}}$ and finally sum over $h$ from 1 to $N$. Then, by setting $v=|D u|^{2}$, we obtain

$$
\begin{aligned}
0 & =a_{i j} D_{i j h} u D_{h} u+D_{h} a_{i j} D_{i j} u D_{h} u+D_{z} a_{i j} D_{i j} u\left(D_{h} u\right)^{2}+D_{p_{s}} a_{i j} D_{i j} u D_{h s} u D_{h} u \\
& +D_{h} a D_{h} u+D_{z} a\left(D_{h} u\right)^{2}+D_{p_{s}} a D_{h s} u D_{h} u-D_{h t} u D_{h} u \\
& =\frac{1}{2} a_{i j} D_{i j} v-a_{i j} D_{h i} u D_{h j} u+D_{h} a_{i j} D_{i j} u D_{h} u+D_{z} a_{i j} D_{i j} u\left(D_{h} u\right)^{2} \\
& +\frac{1}{2}\left(D_{p_{s}} a_{i j} D_{i j} u+D_{p_{s}} a\right) D_{s} v+D_{h} a D_{h} u+D_{z} a\left(D_{h} u\right)^{2}-\frac{1}{2} v_{t} .
\end{aligned}
$$


Therefore, by the Cauchy-Schwartz inequality, we estimate the terms involving the derivatives $D_{x} a_{i j}$ and $D_{z} a_{i j}$, and we obtain, for every $\delta>0$,

$$
\begin{aligned}
& \frac{1}{2}\left(a_{i j} D_{i j} v+\left(D_{p_{s}} a_{i j} D_{i j} u+D_{p_{s}} a\right) D_{s} v-v_{t}\right) \\
& \quad+|D a| \sqrt{v}+\left(D_{z} a+\frac{N^{2}}{2 \delta}\right) v+\frac{N^{2}}{2 \delta} v^{2}+F \geq 0
\end{aligned}
$$

where

$$
F=-a_{i j} D_{i h} u D_{j h} u+\delta\left|D_{x, z} a_{i j}\right|^{2}\left(D_{i j} u\right)^{2} .
$$

Aiming to apply Theorem 1.1 to (2.6), we estimate the term $F$ by means of condition (H.1) or (H.2).

Under condition (H.1) and by choosing $\delta=1$, we have

$$
F=-a_{i j} D_{i h} u D_{j h} u+\left|D_{x, z} c_{j}\right|^{2} v_{j}^{2} \leq\left|D_{x, z} c_{j}\right|^{2} v_{j}^{2} .
$$

Then we can rewrite (2.6) in the form

$$
a_{i j}(X, v, D v) D_{i j} v+b(X, v, D v)-v_{t} \geq 0
$$

with $b$ such that

$$
b(X, v, 0)=2|D a| \sqrt{v}+\left(2 D_{z} a+N^{2}\right) v+N^{2} v^{2} .
$$

The thesis follows by Theorem 1.1 since by assumption $b(X, v, 0)$ is bounded on $Q_{T} \times K$ for every $K$ bounded subset of $\mathbb{R}$.

Conversely, under assumption (H.2), we can choose a positive $\delta=\delta\left(k, M, D_{x, z} a_{i i}\right)$ sufficiently small so that

$$
F=-a_{i i}\left(D_{i h} u\right)^{2}+\delta\left|D_{x, z} a_{i i}\right|^{2}\left(D_{i i} u\right)^{2} \leq\left(-a_{i i}+\delta\left|D_{x, z} a_{i i}\right|^{2}\right)\left(D_{i i} u\right)^{2} \leq 0 .
$$

Indeed, if (H.2) holds and $a_{i i}(X, z, p) \geq k$, then we set $\delta>0$ so that

$$
-a_{i i}+\delta\left|D_{x, z} a_{i i}\right|^{2} \leq-k+\delta\left|D_{x, z} a_{i i}\right|^{2} \leq 0 .
$$

On the other hand, if $a_{i i}(X, z, p) \leq k$ and $M \delta \leq 1$, by (1.8), we get

$$
-a_{i i}+\delta\left|D_{x, z} a_{i i}\right|^{2} \leq a_{i i}(-1+\delta M) \leq 0 .
$$

The thesis follows at once, again by Theorem 1.1

Remark 2.1. The assumption on the $C^{3}$ regularity of the solution can be dropped if we have some uniform boundary gradient estimates. Indeed, let us assume that $a_{i j}, a \in C^{1, \alpha}$ and there exists a positive constant $C$ such that

$$
\limsup _{\mathcal{P} Q_{T}}\left|D u^{\varepsilon}\right| \leq C,
$$

for every $u^{\varepsilon}$ classical solution of the regularized equation $P_{\varepsilon} u^{\varepsilon}=0$ in $Q_{T}$ (cf. (1.2)). Then estimate (1.9) holds uniformly on $\varepsilon$ and we get the thesis passing at limit. 


\section{REFERENCES}

1. F. Antonelli, A. Pascucci, On the viscosity solutions of a stochastic differential utility problem, to appear in J. Differential Equations.

2. G. BARLES, A weak Bernstein method for fully non-linear elliptic equations, Differential Integral Equations 4, No.2, (1991), 241-262. MR 91k:35087

3. S. Bernstein, Sur la généralisation du probléme de Dirichlet, I, Math. Ann., 62, (1906), 253-271.

4. G. Citti, A. PAscucci, S. Polidoro, Regularity properties of viscosity solutions of a non-Hörmander degenerate equation, J. Math. Pures Appl., 80-9, (2001), 901-918. MR 2002j:35055

5. G. Citti, M. Manfredini, A degenerate parabolic equation arising in image processing, to appear in Commun. Appl. Anal.

6. M. Escobedo, J.L. VAzquez, E. ZuAzuA, Entropy solutions for diffusion-convection equations with partial diffusivity, Trans. Amer. Math. Soc. 343, No.2, (1994), 829-842. MR 94h:35131

7. D. Gilbarg, N.S. Trudinger, Elliptic partial differential equations of second order, SpringerVerlag, Berlin, (2001). MR 2001k:35004

8. G. Huisken, Non-parametric mean curvature evolution with boundary conditions, J. Differential Equations 77, No.2, (1989), 369-378. MR 90g:35050

9. A.V. Ivanov, Quasilinear degenerate and nonuniformly elliptic and parabolic equations of second order, Proceedings of the Steklov Institute of Mathematics, Issue 1 (Russian Vol. 160). Providence, Rhode Island: American Mathematical Society. XI, (1984). MR 85e:35055

10. O.A. Ladyzhenskaya, N.N. Ural'tseva, A boundary value problem for linear and quasilinear parabolic equations I, II, (Russian, English) Am. Math. Soc., Transl., II. Ser. 47, 217-299 (1965); translation from Izv. Akad. Nauk SSSR, Ser. Mat. 26, 5-52, 753-780 (1962).

11. O.A. LADYZHENSKAYA, N.N. URAL'TSEVA, Linear and quasi-linear equations of parabolic type, Transl. Math. Monographs 23. Providence, RI: American Mathematical Society (1968).

12. G.M. Lieberman, Second order parabolic differential equations, Singapore, World Scientific, (1996). MR 98k:35003

13. G.M. Lieberman, Gradient estimates for a new class of degenerate elliptic and parabolic equations, Ann. Scuola Norm. Sup. Pisa Cl. Sci. (4) 21, no. 4, 497-522 (1994). MR 96c:35024

14. A. Pascucci, S. Polidoro, On the Cauchy problem for a nonlinear ultraparabolic equation, preprint

15. J. SerRin, Gradient estimates for solutions of nonlinear elliptic and parabolic equations, "Contributions to Nonlinear Functional Analysis", Proc. Sympos. Univ. Wisconsin, Madison 1971, 565-601. MR 53:6095

Dipartimento di Matematica, Università di Bologna, Piazza di Porta S. Donato 5, 40126 Bologna, ItTALY

E-mail address: manfredi@dm.unibo.it

Dipartimento di Matematica, Università di Bologna, Piazza di Porta S. Donato 5, 40126 Bologna, ITALY

E-mail address: pascucci@dm.unibo.it 